\title{
Papers
}

\section{Skin biopsy rates and incidence of melanoma: population based ecological study}

H Gilbert Welch, Steven Woloshin, Lisa M Schwartz

\begin{abstract}
Objectives To describe changes in skin biopsy rates and to determine their relation with changes in the incidence of melanoma.

Design Population based ecological study.

Setting Nine geographical areas of the United States.

Participants Participants of the Surveillance Epidemiology and End Results (SEER) programme aged 65 and older.

Main outcome measures For the period 1986 to 2001, annual skin biopsy rates for each surveillance area from Medicare claims and incidence rates for melanoma for the same population.

Results Between 1986 and 2001 the average biopsy rate across the nine participating areas increased 2.5-fold among people aged 65 and older (2847 to 7222 per 100000 population). Over the same period the average incidence of melanoma increased 2.4-fold (45 to 108 per 100000 population).

Assuming that the occurrence of true disease was constant, the extra number of melanoma cases that were diagnosed after carrying out 1000 additional biopsies was 12.6 (95\% confidence interval 11.2 to 14.0). After controlling for a potential increase in the true occurrence of disease, 1000 additional biopsies were still associated with 6.9 (3.1 to 10.8) extra melanoma cases diagnosed. Stage specific analyses suggested that 1000 biopsies were associated with 4.4 (2.1 to 6.8 ) extra cases of in situ melanoma diagnosed and 2.3 (0.0 to 4.6) extra cases of local melanoma, but not with the incidence of advanced melanoma. Mortality from melanoma changed little during the period. Conclusion The incidence of melanoma is associated with biopsy rates. That the extra cases diagnosed were confined to early stage cancer while mortality remained stable suggests overdiagnosis - the increased incidence being largely the result of increased diagnostic scrutiny and not an increase in the incidence of disease.
\end{abstract}

The incidence of melanoma of the skin is rising faster than any other major cancer in the United States. In 2002-the most recent year of data-the incidence was about six times that in 1950 .

Some dermatologists suggest that this rising incidence may be more apparent than real $^{1-3}$ and acknowledge that diagnosis on the basis of histology is malleable; studies have shown that pathologists examining the same skin biopsy samples often disagree on the diagnosis of melanoma. ${ }^{4-6}$ Dermatologists argue that some lesions that appear malignant to pathologists are biologically benign-an idea supported by data showing that most of the increased incidence is confined to early stage disease. ${ }^{137}$ Finally, whenever physicians look more closely for melanoma, they find more cases. ${ }^{7-10}$

Population based data have not been reported on skin biopsies, the critical end point of surveillance. We examined data from Medicare, a nationwide health insurance for older Americans, to determine whether changes in the biopsy rate relate to the incidence of melanoma.

\section{Methods}

We used Medicare claims to obtain annual population based rates of skin biopsy for patients aged 65 and older in each of the nine geographical areas included in the US National Cancer Institute's Surveillance Epidemiology and End Results (SEER) programme from 1986 to 2001. We calculated the biopsy rates for each of 14 years (claims were unavailable for 1991 and 1992). To obtain the annual incidence of melanoma for the same population, we used the programme's statistical software (SEER*Stat, version 5.3.0). ${ }^{11}$ We obtained stage specific incidence rates using the surveillance programme's four histological disease stages (in situ, local, regional, and distant) and summed them to produce an incidence rate for all stages combined. Using SEER*Stat's incidence based mortality method we also calculated melanoma incidence and disease specific mortality for all nine geographical areas combined.

\section{Analysis}

We used multiple linear regression to explore the relation between biopsy rate (independent variable) and melanoma rate (dependent variable). The unit of analysis was the surveillance programme's area in an individual year (nine areas, 14 years, 126 observations). To control for regional differences that may affect incidence (for example, latitude, racial composition, practice style) we included an indicator variable for area in all analyses. Our baseline analysis predicts the effect of 1000 additional biopsies on the number of melanoma diagnoses. The implicit assumption is that the true occurrence of disease may differ across areas but that it does not change over time. Our second analysis predicts the effect of biopsy assuming the true occurrence of disease has increased. To do this we used interaction terms for year and area, which allow the incidence of melanoma in each area to increase independently-that is, to have its own slope. Finally, we carried out four stage specific regressions using the same interaction terms when the dependent variable was the incidence of a specific disease stage. All

Detailed model outputs are on bmj.com 
analyses were carried out using Stata 7.0 (see bmj.com for detailed model outputs).

\section{Results}

The incidence of melanoma in older people ( $\geq 65$ years) in nine geographical areas of the US National Cancer Institute's Surveillance Epidemiology and End Results programme showed a steady, striking increase between 1986 and 2001 (fig 1). Most of the increase was in early stage disease (in situ and local) and not late stage disease (regional and distant). Mortality from melanoma changed little during the period.

\section{Relation between biopsy and incidence}

Between 1986 and 2001 the average biopsy rate across the nine areas increased 2.5-fold, from 2847 to 7222 per 100000 population. Over this time the average incidence of melanoma increased 2.4-fold, from 45 to 108 per 100000 population. Figure 2 shows the annual skin biopsy rate and incidence of melanoma for each area during the 14 years, illustrating a positive linear relation.

Table 1 provides estimates for the extra number of cases of melanoma diagnosed in association with an additional 1000 biopsies. Assuming no change in the true occurrence of disease, an additional 1000 biopsies resulted in 12.6 (95\% confidence interval 11.2 to 14.0) number of cases of melanoma. Assuming the true occurrence of disease increased, 1000 additional biopsies were associated with an extra 6.9 (3.1 to 10.8) cases of melanoma. Stage specific analyses suggested that 1000 biopsies were associated with 4.4 (2.1 to 6.8 ) extra cases of in situ
Table 1 Estimate of extra cases of melanoma diagnosed per 1000 additional biopsies by stage of disease

\begin{tabular}{llcc} 
Stage of disease & $\begin{array}{c}\text { Assumption on } \\
\text { incidence of disease }\end{array}$ & $\begin{array}{c}\text { Extra cases diagnosed } \\
\text { per } \mathbf{1 0 0 0} \text { biopsies } \\
\mathbf{( 9 5 \%} \mathbf{~ C l})\end{array}$ & P value \\
\hline All stages & No change & $12.6(11.2$ to 14.0$)$ & $<0.001$ \\
\hline All stages & Increase & $6.9(3.1$ to 10.8$)$ & $<0.001$ \\
\hline Stage specific: & & & \\
\hline In situ & Increase & $4.4(2.1$ to 6.8$)$ & $<0.001$ \\
\hline Local & Increase & $2.3(0.0$ to 4.6$)$ & 0.05 \\
\hline Regional & Increase & $0.3(-0.5$ to 1.1$)$ & 0.45 \\
\hline Distant & Increase & $-0.1(-0.6$ to 0.4$)$ & 0.68 \\
\hline
\end{tabular}

melanoma and 2.3 (0.0 to 4.6) extra cases of local melanoma, but not with the incidence of advanced melanoma.

\section{Discussion}

Skin biopsy rates have increased substantially in each of the nine geographical areas participating in the US National Cancer Institute's Surveillance Epidemiology and End Results programme. This growth is associated with an increased rate of melanoma detection-a finding that persisted even after assuming an increase in the true occurrence of disease. From stage specific analyses we found that this relation was confined to local and in situ melanoma and was not evident in advanced disease. This finding supports the idea that the increased incidence of melanoma is largely the result of increased diagnostic scrutinythat is, skin lesions are being biopsied that would not have been in the past.

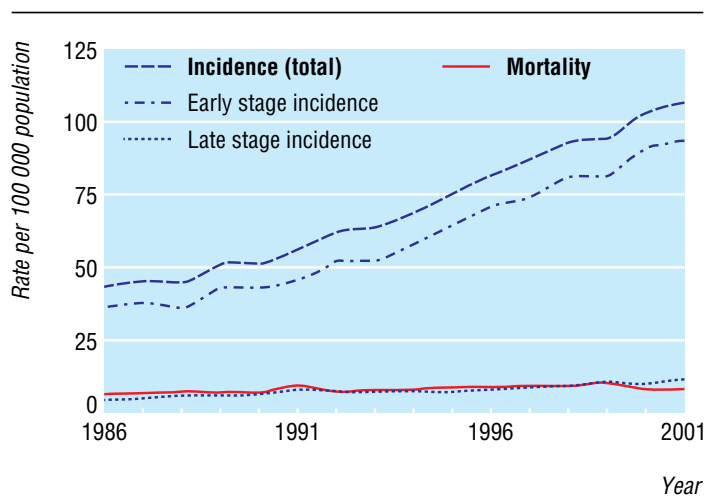

Fig 1 Incidence of melanoma and mortality in population aged 65 and older residing in one of nine US areas participating in Surveillance Epidemiology and End Results programme, 1986-2001. Early stage refers to in situ and local disease; late stage refers to regional and distant disease

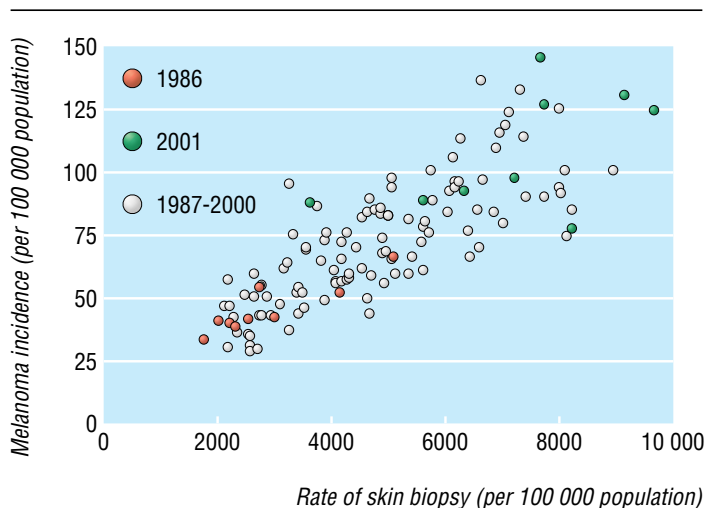

Fig 2 Scatterplot of annual rate of skin biopsy and incidence of melanoma for residents age 65 and older in each of nine US areas participating in Surveillance Epidemiology and End Results programme, 1986-2001 
Table 2 Expected change in disease stage incidence and mortality given competing explanations for apparent rise in cancer incidence

\begin{tabular}{|c|c|c|c|}
\hline \multirow{2}{*}{$\begin{array}{l}\text { Explanations for } \\
\text { apparent rise in } \\
\text { incidence }\end{array}$} & \multicolumn{2}{|c|}{ Expected change in incidence } & \multirow{2}{*}{$\begin{array}{l}\text { Expected change } \\
\text { in mortality }\end{array}$} \\
\hline & Early stage disease & Late stage disease & \\
\hline $\begin{array}{l}\text { Increased diagnostic } \\
\text { scrutiny, no change in } \\
\text { true occurrence of } \\
\text { disease }\end{array}$ & Increase & No change & No change \\
\hline $\begin{array}{l}\text { Increased true } \\
\text { occurrence of disease }\end{array}$ & Increase & Increase & Increase \\
\hline
\end{tabular}

Our data for trend also suggest that the true occurrence of melanoma has not changed. Table 2 outlines the expected change in stage specific incidence and mortality given different explanations for an apparent rise in incidence. The incidence of early stage disease has risen rapidly whereas the incidence for late stage disease and mortality have been relatively stable (fig 1), findings arguing against a dramatic increase in the true occurrence of disease. The predominant explanation for the apparent rise in melanoma incidence is instead overdiagnosis, the result of increased diagnostic scrutiny.

Some might posit an alternative explanation, arguing that stable mortality in the face of rising incidence reflects improved melanoma therapy. These improvements might be the net effect of two factors: starting treatment at earlier stages of disease, or better treatment for a given stage. But this seems implausible. For it to be true, the improvements afforded by early diagnosis and better treatment would need to exactly match the pace of the underlying increase in disease burden; they cannot be too slow or mortality would rise, nor can they be too fast or mortality would decrease.

The finding that the extent to which cancer is identified seems to be directly related to how closely it is looked for has been observed with several cancers, including lung cancer, ${ }^{12}$ breast cancer, ${ }^{13}$ renal cell carcinoma, ${ }^{14}$ and neuroblastoma. ${ }^{15}{ }^{16}$ Perhaps the most powerful example is prostate cancer, where the number of cancers detected is directly related to how aggressively urologists biopsy the prostate. Historically, six needle biopsies were done but now many advocate 12 or more, noting that the more biopsy samples that are taken, the more likely cancer is to be found. ${ }^{17-20}$ Some even advocate "saturation biopsy" (32-38 needle biopsies), as cancers can still be found microscopically in men who were cancer free on the basis of three or more prior biopsies. ${ }^{21}$

Our analysis has several limitations. Firstly, the data only represent people aged 65 and older. We do not know whether biopsy rates have changed in younger people or how any change might relate to the incidence of melanoma. Secondly, our biopsy rates include biopsies for lesions that were not considered to be related to melanoma before the biopsy, but rather basal or squamous cell carcinoma or a cutaneous manifestation of systemic disease. This concern is highlighted by biopsy rates that are nearly two orders of magnitude higher than melanoma incidence. Such imprecision in measuring exposure, however, only biases our results to the null.

Thirdly, some might question how we modelled an increase in the true occurrence of disease. Our analysis considers the independent effect of an area's biopsy rate on its melanoma rate if each area is allowed its own initial melanoma rate (an area specific y intercept) and its own melanoma growth rate (an area specific slope). By including a term for time to control for melanoma growth rate, we may have controlled for factors besides changes in true disease occurrence. Aspects of diagnostic scrutiny other than biopsy rate may also change over time, particularly the pro-

\section{What is already known on this topic}

Some dermatologists suspect that the rise in incidence of melanoma may reflect more skin biopsies, not more disease

\section{What this study adds}

The increasing incidence of melanoma between 1986 and 2001 in the United States is closely associated with biopsy rates

The increased incidence was mostly in early stage disease; yet mortality from melanoma remained stable

This pattern suggests overdiagnosis, largely as a result of increased diagnostic scrutiny and not an increase in the true occurrence of disease

portion of biopsies carried out for pigmented lesions. Although we are unable to estimate precisely the true effect of biopsy rate, we were able to bound the estimate by carrying out analyses after assuming both increasing and stable true occurrence of disease (with and without time). Thus the true effect of 1000 additional biopsies is likely to be between 6.9 and 12.6 extra cases of melanoma diagnosed.

Finally, our investigation shares the limitation of all observational research in that although we show an association between biopsy rate and incidence, inferences about causation are more speculative. A causal relation is suggested, however, because the relation is confined to the category of disease (early stage melanoma) that would be expected to be related to diagnostic scrutiny. Furthermore, the finding of stable mortality in this population suggests that the bulk of these additional cases of melanoma may appear malignant on histology but are nonetheless biologically benign.

We thank Elliott Fisher for his guidance and help in obtaining claim data from Medicare, Weiping Zhou for analysing the claims, and Jennifer A Snide for analysing the surveillance data.

Contributors: HGW analysed the data and wrote the first draft. SW and LMS participated in the design of the analyses and made important contributions to the presentation of the work. HGW is the guarantor. The views expressed do not necessarily represent those of the Department of Veterans Affairs or the United States government.

Funding: This study was supported in part by a research enhancement award from the Department of Veterans Affairs (03-098) and with a grant from the National Institute of Aging (PO1 AG19783-01). LMS is supported by Veterans Affairs career development awards in health services research and development. LMS and SW are supported by the generalist physician faculty scholars programme of the Robert Wood Johnson Foundation.

Competing interests: None declared.

Ethical approval: Dartmouth institutional review board.

1 Swerlick RA, Chen S. The melanoma epidemic: more apparent than real? Mayo Clin Proc 1997;72:559-64.

Florez A Cruces M. Melanoma epidemic: true or false? Int J Dermatol 2004:43:405-7.

3 Beddingfield FC. The melanoma epidemic: res ipsa loquitur. Oncologist 2003;8:459-65.

4 Farmer ER, Gonin R, Hanna MP. Discordance in the histopathologic diagnosis of melanoma and melanocytic nevi between expert pathologists. Hum Pathol 1996;27:528-31

5 Corona R, Mele A, Amini M, De Rosa G, Coppola G, Piccardi P, et al. Interobserver variability on the histopathologic diagnosis of cutaneous melanoma and other pigmented skin lesions. J Clin Oncol 1996;14:1218-23.

6 Weinstock MA, Barnhill RL, Rhodes AR, Brodsky GL. Reliability of the histopathologic diagnosis of melanocytic dysplasia. Arch Dermatol 1997;133:953-8.

Burton RC, Armstrong BK. Recent incidence trends imply a nonmetastasizing form of invasive melanoma. Melanoma Res 1994;4:107-13.

8 Hiatt RA, Fireman B. The possible effect of increased surveillance on the incidence of Hiat RA, Firnan B. The possible efrect of inc

Burton RC, Coates MS, Hersey P, Roberts G, Chetty MP, Chen S, et al. An analysis of a Burton RC, Coates MS, Hersey P, Roberts G, Che
melanoma epidemic. Int J Cancer 1993;55:765-70.

10 Swerlick RA, Chen S. The melanoma epidemic. Is increased surveillance the solution or the problem? Arch Dermatol 1996;132:881-4. 
11 Surveillance research program. National Cancer Institute SEER*Stat software, version 5.3.0. www.seer.cancer.gov/seerstat (accessed 31 Aug 2004).

12 Sone S, Takashima S, Li F, Yang Z, Honda T, Maruyama Y, et al. Mass screening for lung cancer with mobile spiral computed tomography scanner. Lancet 1998;351:1242-5.

13 Zahl PH, Strand BH, Maehlen J. Incidence of breast cancer in Norway and Sweden during introduction of nationwide screening: prospective cohort study. $B M J$ 2004;328:921-4.

14 Spouge AR, Wilson SR, Wooley B. Abdominal sonography in asymptomatic executives: prevalence of pathologic findings, potential benefits, and problems. J Ultrasound Med 1996;15:763-7.

15 Woods WG, Gao RN, Shuster JJ, Robison LL, Bernstein M, Weitzman S, et al. Screening of infants and mortality due to neuroblastoma. N Engl J Med 2002;346:1041-6.

16 Schilling FH, Spix C, Berthold F, Erttmann R, Fehse N, Hero B, et al. Neuroblastoma Schilling FH, Spix C, Berthold F, Erttmann R, Fehse N, Hero
screening at one year of age. $N$ Engl J Med 2002;346:1047-53.

17 Eskew LA, Bare RL, McCullough DL. Systematic 5 region prostate biopsy is superior to sextant method for diagnosing carcinoma of the prostate. J Urol 1997;157:199-202.

18 Babaian RJ, Toi A, Kamoi K, Troncoso P, Sweet J, Evans R, et al. A comparative analysis of sextant and an extended 11-core multisite directed biopsy strategy. $J$ Urol 2000;163:152-7.

19 Arnold PM, Niemann TH, Bahnson RR. Extended sector biopsy for detection of carcinoma of the prostate. Urol Oncol 2001;6:91-3.
20 Durkan GC, Sheikh N, Johnson P, Hildreth AJ, Greene DR. Improving prostate cancer detection with an extended-core transrectal ultrasonography-guided prostate biopsy protocol. BrJ Urol Int 2002;89:33-9.

21 Fleshner N, Klotz L. Role of "saturation biopsy" in the detection of prostate cancer among difficult diagnostic cases. Urology 2002;60:93-7.

(Accepted 7 June 2005)

doi 10.1136/bmj.38516.649537.E0

VA Outcomes Group, Department of Veterans Affairs Medical Center, White River Junction, VT 05009, USA

H Gilbert Welch professor of medicine

Steven Woloshin associate professor of medicine

Lisa M Schwartz associate professor of medicine

Correspondence to: $\mathrm{H}$ Gilbert Welch, Center for the Evaluative Clinical Sciences,

Dartmouth Medical School, Hanover, NH 03755-1404, USA

H.Gilbert.Welch@dartmouth.edu 